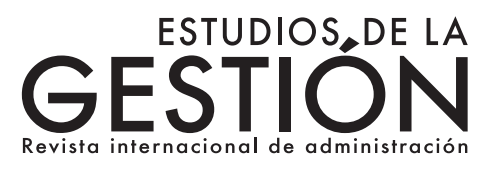

\title{
El capital humano y los retornos a la educación en Ecuador
}

\author{
Edisson Tarupi Montenegro \\ Universidad de los Andes (Colombia) \\ armand0137@hotmail.com
}

Fecha de presentación: 4 de enero de 2015 • Fecha de aceptación: 20 de octubre de 2015

Artículo de investigación 


\section{Resumen}

Este trabajo analiza los retornos a la educación en el Ecuador en donde el mercado laboral reconoce la escolaridad de los empleados y, por lo tanto, se requiere establecer las variaciones salariales debidas a la educación y sus niveles. Los retornos a la educación secundaria caen mientras en la educación superior presentan una tendencia creciente.

También se presentan indicadores generales de la variación de los salarios que permiten identificar las tendencias por regiones y por sexo.

Palabras clave: capital humano, ingresos, retornos, educación, hogares, Ecuador.

JEL: J24 Capital humano, cualificación, elección de ocupación, productividad del trabajo; J31 Nivel y estructura salarial, diferencias salariales por cualificación, formación, ocupación.

\section{Abstract}

This paper analyzes the returns to education in Ecuador where the labor market recognizes the level of education in employees and therefore requires setting wage variations due to education and levels, finding that returns to secondary education fall while higher education show a growing trend.

General indicators of the variation in wages that identify trends by region and gender are also presented.

Keywords: Human capital, income, returns, education, homes, Ecuador.

JEL: J24 Human capital, qualifications, choice of occupation, labor productivity; J31 Level and salary structure, qualification pay gap, training, employment.

\section{Resumo}

Este artigo analisa os retornos da educação no Equador, onde o mercado de trabalho reconhece os funcionários de escolaridade e, portanto, é necessário para estabelecer os aumentos salariais decorrentes de níveis de educação, descoberta que retorna à queda ensino secundário enquanto ensino superior mostram uma tendência crescente.

Também são apresentados indicadores gerais de mudanças nos salários que identificam tendências por região e sexo.

Palavras-chave: capital humano, renda, retornos, educação, casas, Equador.

JEL: J24 Capital humano, qualificações, Escolha da profissão, A produtividade do trabalho; J31 Nível ea estrutura salarial, disparidades salariais cualifación, formação, emprego. 


\section{Introducción}

E 1 presente trabajo se apoya en la literatura de los retornos a la educación y rescata la importancia de invertir en capital humano, en el desarrollo de competencias y habilidades con el propósito de mejorar la eficiencia productiva, lo que, a decir de Schultz (1961), mediante la escolaridad formal, capacitación y demás proyectos educativos se fomenta el desarrollo social, productivo y ambiental, y se destaca como principal factor a la educación, sea general o específica, que reclama mayor atención y apoyo en países como Ecuador. De ahí que se rescata el escenario educativo ecuatoriano con el propósito de sugerir implicaciones de política que mejoren la productividad y, de esta manera, reconsiderar aquellas políticas sociales, en especial las educativas que contribuyan a la calidad de vida y al buen vivir.

Cabe resaltar las dimensiones del desarrollo humano en las cuales se destaca la escolaridad y los niveles educativos que también juegan un rol significativo en la perspectiva del capital humano y su incidencia en el bienestar social (Becker 1964). En este sentido se trata de explicar cómo la inversión en educación influye en los diferentes niveles salariales que se identifican en el mercado laboral ecuatoriano, mediante la aplicación del modelo de capital humano a la Mincer (1958), que permite identificar los efectos de los distintos niveles de educación, de experiencia, edad, sexo, entre otras características sobre los ingresos.

Para comprender los diferentes resultados de diversos autores, en cuanto a la estimación de los retornos a la educación, se consideran los modelos y especificaciones que corrigen errores de selección y correlaciones al calcular el retorno a la educación según el método de mínimos cuadrados ordinarios (OLS) (Heckman 1979).

Así, la posible correlación se corrige identificando un conjunto de variables instrumentales que afecten la escolaridad pero que no afecten el salario. 
O la inclusión de variables relevantes, por ejemplo, el trimestre de nacimiento utilizados por Angrist y Krueger (1991) como variable instrumental para la educación, cuyo resultado confirma que las personas nacidas durante el primer trimestre poseen un nivel menor de educación que aquellas personas que nacen en los siguientes trimestres.

Para otro trabajo, Angrist y Krueger (1992) usaron un procedimiento diferente basado en la asignación del número de la lotería durante la guerra de Vietnam como variable instrumental, con lo cual los alumnos matriculados pueden excluirse del servicio militar, fundamentalmente aquellos que están en edad de reclutarse.

Por su parte, Butcher y Case (1994) analizan la composición del efecto de hermanos en el logro educacional. Ya que el sexo de los chicos es aleatorio, la composición del sexo en la familia de tamaño dado es aleatoria. Se ofreció evidencia de que mujeres con una o más hermanas tienen significativamente menor educación que aquellas mujeres que tienen hermanos. Ellos utilizan una variable que detecta la presencia de hermanas en el hogar como variable instrumental para la educación en una ecuación de salarios, incluyendo controles por tamaño familiar. A pesar de que la estimación del retorno a la educación es imprecisa, esta es muy superior al encontrado con métodos tradicionales.

En cambio, Card (1999) usa un indicador simple como variable de control: la presencia de establecimientos educativos en las cercanías del hogar. Las personas que crecen cerca de un colegio tienen significativamente mayor educación y, por lo tanto, más ingresos que otras personas. Cuando se utiliza la proximidad al colegio, se obtienen estimadores con el $80 \%$ más alto a los obtenidos por OLS. Al utilizar una especificación alternativa que considera la educación de los padres se obtiene un estimador con un 30\% superior al OLS.

Además se destaca la alternativa en el intento de eliminar el sesgo, que se presume se da al estimar por OLS, que es el de efectos fijos en el que se obtuvo un retorno en un 28\% mayor que por el método de efectos fijos (Griliches 1977). Por su parte, Angrist y Newey (1991) suponen que la existencia de correlación entre componentes de los ingresos y la educación se debe a elementos que no dependen del tiempo, por lo que los estimadores serán consistentes. Encuentran que el retorno a la educación se subestima al estimarlo mediante OLS.

Utilizando datos para mellizos idénticos encuestados simultáneamente con un supuesto similar al de Angrist y Newey (1991), Ashenfelter y Krueger 
(1994) suponen que los errores de la educación y los salarios se correlacionan por efectos familiares, ya que puede darse que, al menos, una pareja de mellizos tenga diferente nivel de educación, y, por tanto, encuentran un mayor retorno a la educación.

En este contexto es muy importante el trabajo realizado para Chile por Contreras, Bravo y Medrano (1999) en el cual se estima el retorno a la educación en su forma tradicional minceriana, para luego incorporar un considerable número de variables instrumentales que permiten corregir tanto la omisión de variables y el sesgo al seleccionar la representatividad en la muestra; obtienen que los signos de los parámetros son consistentes con la teoría que sostiene que la educación y la experiencia tienen efectos positivos sobre el nivel de ingresos, si bien la experiencia presenta retornos decrecientes. Proporciona resultados efectivos sobre la tasa de crecimiento que sufre el ingreso debido al efecto educación especialmente superior.

En sus trabajos, Psacharopoulos (1980 y 1985) analiza el hecho de cuán beneficioso es invertir en educación, para lo cual realiza estimaciones aplicando varias técnicas de las cuales la más relevante para este trabajo es el método de la estimación de la función de ingreso realizada para Venezuela y Guatemala, obteniendo que la tasa de retorno de la educación de invertir en mujeres es mayor que la correspondiente para los hombres y también reporta un nivel alto en la tasa de retorno de la primaria explicando que se debe al hecho de que una tercera parte de la fuerza de trabajo no tiene ninguna educación, por lo que tienen un pago alto en el margen cuando alguno completa el nivel primario.

Ya en el contexto ecuatoriano, Samaniego (1995) hace referencia a la importancia de la experiencia en la función de ingreso. Elabora una interpretación al estilo McKinnon, en la cual los ingresos individuales tienen dos restricciones: la instrucción, es decir, el máximo de ingreso posible en el tiempo, en un primer momento, está condicionado por el nivel de instrucción adquirido; pero, después de cierto período laboral, el máximo de ingresos no se obtiene ya en función del nivel de instrucción, sino del nivel de experiencia adquirida. Señala que desde una perspectiva económica el mercado laboral ecuatoriano presenta imperfecciones significativas en cuanto a sensibilidad de los ingresos-nivel de instrucción.

Otro aspecto de rescatar en Samaniego (1995) se presenta cuando se hace alusión a la amplia existencia de lo que Lam y Schoeni (1993) llaman ne- 
potismo en un estudio para Brasil, o conexiones familiares, perdiéndose la característica de un mercado laboral competitivo, lo que refuerza el hecho de que las conexiones familiares pesan más que la escolaridad en el ingreso en el momento de elegir un trabajador.

Otro hecho que evidencia Mancero (1997) es que, dentro de la estructura productiva ecuatoriana, hay actividades económicas en las que una escasa calificación laboral basta y sobra para percibir ingresos superiores a los que se podría obtener con más años de esfuerzo educativo. Este es el caso del comercio, cuyo retorno por sector es más alto, aun con bajos niveles educativos. En este contexto y con los resultados obtenidos podemos concluir que una mayor preparación contribuye significativamente a mejorar los ingresos.

En el caso chileno, Bravo y Marinovic (1997) también utilizan una descomposición simple de varianza con los resultados de los retornos y encuentran que aproximadamente dos tercios del incremento en la desigualdad salarial registrado en Chile entre 1974 y 1987 puede ser atribuido a factores observables (educación y experiencia potencial).

En una aproximación de tipo no-paramétrica, concentrando la atención en características demográficas de la población, Katz y Murphy (1992) analizan la estructura salarial de Estados Unidos entre 1963 y 1987. Muestran que el importante crecimiento en la demanda por trabajadores más educados, más calificados y trabajadores de sexo femenino puede ser determinante en los cambios observados en la estructura salarial.

Finalmente, en el trabajo realizado por Bravo, Contreras y Rau (1999), en una aproximación no-paramétrica, analiza la evolución de la desigualdad salarial en Chile y los cambios en la demanda relativa de trabajo. Encuentra que la distribución de los salarios se ha mantenido estable en el período de estudio a pesar de los cambios reales y relativos para las diferentes categorías de trabajadores. Estos cambios se comportan de manera diferente para hombres y mujeres, según distintas categorías de educación, prevaleciendo un patrón común para la categoría de educación universitaria en la cual se produce un aumento en la demanda relativa de trabajo.

El análisis de los trabajos revisados evidencia la falta de atención a los temas de educación, ingresos y empleo que se presentan en el país, no obstante también se puede inferir que en el Ecuador, al igual que en otros países, el retorno a la educación es significativo, es decir, afecta en las decisiones 
salariales, más aún cuando se controla por otras variables como: educación de los padres y capacitación, calidad de la educación, a más de las que plantean los estudios analizados.

\section{Objetivo}

El objetivo de esta investigación es encontrar empíricamente los retornos a la educación, midiendo la variación de los salarios según sus determinantes observables (escolaridad y experiencia) e inobservables (educación de los padres, calidad de la educación, entre otros aspectos), para la población masculina ecuatoriana.

\section{Metodología}

Con el enfoque de capital humano se estima el retorno a la educación utilizando la conocida ecuación de Mincer $(1958)^{1}$ que expresa el nivel de salario en función del nivel educativo y de la experiencia del individuo, controlando por otras características que lo identifican, e introduciendo ciertas variaciones. Para obtener los resultados esperados en esta medición, no basta examinar la relación entre educación e ingreso, ya que estas diferencias salariales no son explicadas en su totalidad por la educación, sino que involucran otros factores como: experiencia, experiencia efectiva; y otros factores que segmentan el ingreso y discriminan laboralmente como: la educación de los padres, sector económico, migración, capacitación, sindicalización, entre otros.

La encuesta de condiciones de vida (ECV-95) reporta un conjunto de características del trabajador referentes al contrato de trabajo, el tiempo de trabajo, si repitió un curso, el tipo de establecimientos educacionales a los que asistió, educación del padre, educación de la madre, lugar en donde se capacitó, migración, distancia del hogar al establecimiento educativo, entre otras. Lo cual permite incorporar al análisis diversas variables de control individual y colectivamente.

1. Mincer (1958) asume que la función es lineal con respecto a la educación y exponencial con respecto a la experiencia. A dicha función se le agregan otras variables explicativas. 
Tabla 1

Estadística descriptiva por regiones para Ecuador

\begin{tabular}{|c|c|c|c|c|c|c|c|c|}
\hline \multirow{3}{*}{ Variables } & \multirow{2}{*}{\multicolumn{2}{|c|}{ País }} & \multicolumn{6}{|c|}{ REGIONES } \\
\hline & & & \multicolumn{2}{|c|}{ Costa } & \multicolumn{2}{|c|}{ Sierra } & \multicolumn{2}{|c|}{ Oriente } \\
\hline & Media & Desv.est. & Media & Desv.est. & Media & Desv.est. & Media & Desv.est \\
\hline Edad & 33,88 & 10,64 & 33,73 & 10,29 & 34,12 & 10,96 & 31,82 & 10,45 \\
\hline Salario mensual (dólares) & 272,95 & 311,61 & 274,15 & 317,72 & 275,93 & 312,51 & 190,00 & 108,85 \\
\hline Salario por hora (dólares) & 1,40 & 1,56 & 1,37 & 1,54 & 1,45 & 1,60 & 1,04 & 0,71 \\
\hline Log. Sal / Hora (dólares) & $-0,01$ & 0,80 & $-0,04$ & 0,81 & 0,04 & 0,78 & $-0,19$ & 0,72 \\
\hline Horas & 51,24 & 14,39 & 52,31 & 14,56 & 50,25 & 14,13 & 51,39 & 15,46 \\
\hline Escolaridad & 8,46 & 5,11 & 8,18 & 5,06 & 8,76 & 5,16 & 7,80 & 4,80 \\
\hline Escolaridad $^{2}$ & 97,72 & 103,06 & 92,44 & 99,17 & 103,22 & 106,90 & 83,73 & 89,54 \\
\hline Experiencia efectiva & 6,81 & 7,66 & 6,59 & 7,65 & 7,01 & 7,68 & 6,73 & 7,45 \\
\hline Experiencia efectiva ${ }^{2}$ & 104,94 & 214,73 & 101,82 & 219,91 & 108,01 & 210,83 & 100,37 & 200,60 \\
\hline Experiencia potencial & 19,42 & 11,19 & 19,55 & 10,83 & 19,37 & 11,54 & 18,02 & 10,85 \\
\hline Experiencia potencial $\left.\right|^{2}$ & 502,17 & 546,33 & 499,00 & 515,04 & 508,03 & 575,63 & 441,49 & 505,62 \\
\hline$(E s c-6)^{*} \mathrm{D} 6$ & 3,35 & 4,22 & 3,15 & 4,09 & 3,56 & 4,34 & 2,82 & 3,82 \\
\hline$(E s c-12)^{*} \mathrm{D} 12$ & 0,85 & 2,03 & 0,75 & 1,90 & 0,96 & 2,15 & 0,56 & 1,57 \\
\hline Agricultura & 0,05 & 0,21 & 0,08 & 0,28 & 0,01 & 0,12 & 0,06 & 0,23 \\
\hline Minería & 0,02 & 0,14 & 0,02 & 0,15 & 0,02 & 0,13 & 0,02 & 0,14 \\
\hline Industria & 0,17 & 0,38 & 0,20 & 0,40 & 0,16 & 0,37 & 0,01 & 0,12 \\
\hline Suministros & 0,02 & 0,13 & 0,02 & 0,15 & 0,01 & 0,11 & 0,01 & 0,10 \\
\hline Construcción & 0,04 & 0,19 & 0,02 & 0,15 & 0,05 & 0,21 & 0,08 & 0,27 \\
\hline Comercio & 0,18 & 0,39 & 0,21 & 0,41 & 0,16 & 0,36 & 0,09 & 0,28 \\
\hline Transporte & 0,07 & 0,26 & 0,06 & 0,25 & 0,07 & 0,26 & 0,09 & 0,28 \\
\hline Servicios financieros & 0,08 & 0,27 & 0,07 & 0,26 & 0,09 & 0,28 & 0,06 & 0,24 \\
\hline Servicios comunales & 0,37 & 0,48 & 0,30 & 0,46 & 0,43 & 0,50 & 0,58 & 0,50 \\
\hline Educ. padre primaria & 0,48 & 0,50 & 0,47 & 0,50 & 0,49 & 0,50 & 0,62 & 0,49 \\
\hline Educ. padre secundaria & 0,17 & 0,38 & 0,19 & 0,39 & 0,16 & 0,36 & 0,10 & 0,30 \\
\hline Educ. padre superior & 0,06 & 0,24 & 0,05 & 0,23 & 0,07 & 0,25 & 0,01 & 0,12 \\
\hline Educ. madre primaria & 0,48 & 0,50 & 0,46 & 0,50 & 0,50 & 0,50 & 0,56 & 0,50 \\
\hline Educ. madre secundaria & 0,15 & 0,36 & 0,18 & 0,38 & 0,13 & 0,34 & 0,03 & 0,16 \\
\hline Educ. madre superior & 0,03 & 0,16 & 0,03 & 0,17 & 0,03 & 0,16 & 0,00 & 0,07 \\
\hline Cap. Secap & 0,03 & 0,16 & 0,02 & 0,16 & 0,03 & 0,16 & 0,03 & 0,17 \\
\hline Cap. emp. trabaja & 0,13 & 0,34 & 0,11 & 0,32 & 0,15 & 0,36 & 0,10 & 0,30 \\
\hline Cap. ONG & 0,03 & 0,17 & 0,04 & 0,19 & 0,02 & 0,15 & 0,00 & 0,05 \\
\hline Cap. emp. especial & 0,05 & 0,21 & 0,05 & 0,22 & 0,05 & 0,21 & 0,03 & 0,18 \\
\hline Cap. MEC & 0,02 & 0,15 & 0,03 & 0,16 & 0,02 & 0,13 & 0,07 & 0,26 \\
\hline Cap. inst. oficial & 0,06 & 0,24 & 0,05 & 0,22 & 0,07 & 0,25 & 0,05 & 0,22 \\
\hline Cap. otra institución & 0,02 & 0,14 & 0,01 & 0,11 & 0,03 & 0,16 & 0,00 & 0,06 \\
\hline
\end{tabular}




\begin{tabular}{|l|ll|ll|ll|ll|} 
Dummy contrato & 0,69 & 0,46 & 0,67 & 0,47 & 0,71 & 0,46 & 0,63 & 0,48 \\
Dummy sindicato & 0,21 & 0,41 & 0,18 & 0,38 & 0,24 & 0,43 & 0,35 & 0,48 \\
Dummy est. fiscal & 0,06 & 0,24 & 0,05 & 0,22 & 0,07 & 0,25 & 0,06 & 0,24 \\
Dummy est. f.comicional & 0,00 & 0,03 & 0,00 & 0,00 & 0,00 & 0,04 & 0,01 & 0,10 \\
Dummy est. privado & 0,03 & 0,18 & 0,03 & 0,17 & 0,04 & 0,19 & 0,04 & 0,19 \\
Dummy ed. no presencial & 0,01 & 0,08 & 0,00 & 0,00 & 0,01 & 0,11 & 0,00 & 0,07 \\
Dummy cercanía a est. & 0,04 & 0,20 & 0,04 & 0,20 & 0,04 & 0,20 & 0,07 & 0,26 \\
Dummy repiten año esc. & 0,02 & 0,12 & 0,01 & 0,11 & 0,02 & 0,13 & 0,04 & 0,19 \\
Dummy migración & 0,19 & 0,39 & 0,17 & 0,38 & 0,20 & 0,40 & 0,28 & 0,45 \\
\hline No. de observaciones & \multirow{2}{*}{1093} & \multicolumn{2}{|c|}{416} & & \multicolumn{2}{|c|}{559} & & \multicolumn{3}{|c|}{118} \\
\hline
\end{tabular}

Nota: se consideran hombres obreros y empleados que trabajan jornada completa.

D6: Dummy que toma el valor de 1 si la persona tiene más de 6 años de educación.

D12: Dummy que toma el valor de 1 si la persona tiene más de 12 años de educación.

Las variables monetarias se expresan en dólares de 1995.

Fuente: INEC 1995.

Elaboración propia.

Considerando la disponibilidad de información, primeramente podemos escribir en forma generalizada la ecuación a estimar por OLS.

\section{Modelo 1}

$$
\mathrm{LnWh}=\mathrm{c}+\mathrm{b}_{1} * \operatorname{esc}+\mathrm{b}_{2}^{*} \exp +\mathrm{b}_{3}^{*} \exp ^{2}+\mathrm{b}_{\mathrm{i}} * \mathrm{~S}_{\mathrm{i}}+\mu_{\mathrm{i}}
$$

Donde se asume que (LnWh) es el logaritmo natural del salario por hora, se explica por los años de educación del trabajador (esc), la experiencia y el sector económico en que se desempeña $\left(\mathrm{S}_{\mathrm{i}}\right)$ y finalmente los residuos $\left(\mu_{\mathrm{i}}\right)$. Se espera encontrar un retorno positivo a la educación y la experiencia, si bien se supone que la experiencia genera retorno positivo a tasa decreciente sobre el salario.

Para comprobar si los años de educación presentan un grado de convexidad, se estima el siguiente modelo:

\section{Modelo 2}

$$
\operatorname{LnWh}=\mathrm{c}+\mathrm{b}_{1}{ }^{*} \operatorname{esc}+\mathrm{b}_{2}{ }^{*} \operatorname{esc}^{2}+\mathrm{b}_{3} \exp +\mathrm{b}_{4} * \exp ^{2}+\mathrm{b}_{\mathrm{i}} * \mathrm{~S}_{\mathrm{i}}+\mu_{\mathrm{i}}
$$

En los modelos anteriores se impone un retorno similar para los distintos años de educación, como se trata de estimar un modelo que permita que el retorno entre ciclos educativos se diferencie, planteamos el siguiente modelo: 


\section{Modelo 3}

$\operatorname{LnWh}=\mathrm{c}+\mathrm{b}_{1}{ }^{*} \operatorname{esc}+\mathrm{b}_{2}{ }^{*} \exp +\mathrm{b}_{3}{ }^{*} \exp ^{2}+\mathrm{b}_{4} * \operatorname{Var} 1+\mathrm{b}_{5} * \operatorname{Var} 2+\mathrm{b}_{\mathrm{i}} * \mathrm{~S}_{\mathrm{i}}+\mu_{\mathrm{i}}$ donde:

con:

$\begin{array}{ll}\text { Var } 1=\mathrm{d} 6 *(\text { esc- } 6) & \mathrm{d} 6=1 \text { si esc }>=6,0 \text { en otro caso } \\ \operatorname{Var} 2=\mathrm{d} 12^{*}(\text { esc- } 12) & \mathrm{d} 12=1 \text { si esc }>=12,0 \text { en otro caso } \\ \operatorname{Var} 3=\mathrm{d} 17^{*}(\text { esc- } 17) & \mathrm{d} 17=1 \mathrm{si} \mathrm{esc}>=17,0 \text { en otro caso }\end{array}$

En el modelo anterior se introducen dos dummies que permiten capturar la existencia de distintos retornos a la educación; esto es, para obtener tres tasas de retorno diferentes, que nos darán el retorno de la educación primaria $\left(b_{1}\right)$, educación secundaria $\left(b_{4}\right)$, y educación superior $\left(b_{5}\right)$, permitiendo también comprobar la convexidad del retorno a la educación en el Ecuador.

Al incorporar un modelo menos restrictivo, en el cual se permita que el retorno de cada año sea diferente $\left(d_{j}\right)$, tenemos:

\section{Modelo 4}

$\operatorname{LnWh}=\mathrm{c}+\mathrm{b}_{1} \exp +\mathrm{b}_{2} * \exp ^{2}+\mathrm{Sb}_{\mathrm{j}} * \mathrm{~d}_{\mathrm{j}}+\mathrm{b}_{\mathrm{i}} * \mathrm{~S}_{\mathrm{i}}+\mu_{\mathrm{i}}$

Al estimar esta especificación se incluyen diversas variables de control para comprobar que el retorno a la educación estimado sea confiable, o evaluar si se lo sobre o subestima.

Para evitar el posible sesgo, como primera aproximación, se reestima el retorno a la educación utilizando experiencia efectiva en lugar de experiencia potencial. Luego se introducen controles como: la educación de los padres, capacitación, cercanía al establecimiento educativo, entre otros. Luego se realiza una descomposición simple de varianza de los salarios por hora y una comparación de salarios relativos. Los resultados obtenidos se presentan en la siguiente sección.

\section{Análisis de resultados}

Atendiendo a la especificación planteada y a base de nuestra muestra disponible, al estimar los retornos a la educación, se obtiene la tasa de retorno promedio de la educación básica que fluctúa alrededor del 8 al 9\%. Además se observa 
que el retorno a la educación media es más alto en el Oriente y la Sierra, mientras que el retorno a la educación superior es mayor en la Costa. En general, se evidencia un patrón convexo que se verifica por el hecho del aumento del retorno a la educación superior como sobre todo en la Costa que alcanza un 11,6\%.

Con el objeto de evitar al posible sesgo expuesto al usar la experiencia potencial, se utilizan los datos de experiencia efectiva. Estas estimaciones nos muestran que el retorno a la educación cae $2 \%$, resaltando el hecho de que cuando se utiliza experiencia potencial ${ }^{2}$ se sobrestima el verdadero retorno a la educación.

Al controlar por otras variables no observables tales como educación de los padres, el retorno promedio a la educación se reduce de 6 a 5,7\%, notando que esta caída en el retorno se debe principalmente a una disminución en el retorno de la educación superior, que cae a 10,6\%, mientras la educación media cae de 6,5 a $3,5 \%$.

Por otra parte, al incluir proxies de capacitación, se obtiene que el retorno a la educación cae en un punto porcentual, al igual que cuando incluimos la variable de contrato. Al considerar calidad de la educación a través de la dependencia de los establecimientos educacionales se encuentra que el retorno se reduce en $2 \%$ como cuando se controla por educación de los padres y capacitación de los encuestados.

El menor retorno que podemos encontrar se obtiene introduciendo todas las variables de control disponibles al mismo tiempo, en este caso el retorno promedio de la educación se reduce a $4,5 \%$. No obstante, se rescata que la inversión en educación que cada hogar, familia o individuo hace a su capital humano y en el largo plazo mejora su bienestar vía mejores ingresos y, por lo tanto, un buen vivir digno.

Según los resultados obtenidos podemos concluir que el tipo de educación mayormente compensado con una tasa de retorno de 11,6 es el de la educación superior, como un indicativo de que en el Ecuador se demanda recursos más capacitados, especialmente en estos últimos años, lo cual va acompañado por la expansión y el mejoramiento de los establecimientos universitarios, empleos y empresas más exigentes, por lo cual los empleados y trabajadores calificados requieren un nivel superior, mientras que la educa-

2. Experiencia potencial $=$ edad - experiencia- 6 . 
ción media solo permite observar algunas características del trabajador y no un retorno significativo en sus ingresos.

El análisis de los retornos a la educación es muy importante para los países de América Latina que, en su necesidad de desarrollo, demandan recursos mejor capacitados, reconociendo la educación como generador de capital humano capaz de contribuir al desarrollo de un país. Es necesario implementar políticas de apoyo a la educación, que nos permitan de alguna manera cuantificar y calificar el valor que añade la educación al nivel de ingresos de las personas y, consecuentemente, mejorar el bienestar social.

\section{Conclusiones}

Las estimaciones realizadas para obtener el retorno a la educación permiten formular las siguientes conclusiones: se puede apreciar que la educación influye en los ingresos futuros esperados por los individuos que la reciben, lo que, a su vez, tiene implicaciones en la distribución del ingreso laboral y en el comportamiento de los graduados a lo largo de su vida de trabajo.

En primer lugar, estas proyecciones realizadas para estimar el retorno a la educación entregan los signos de los parámetros consistentes con la teoría que sostiene que la educación y la experiencia tienen efectos positivos sobre el nivel de ingresos, si bien la experiencia presenta retornos a tasas decrecientes.

En cuanto a los perfiles edad/ingresos se comprobó que presentan la forma característica. En todos los grupos educacionales y regionales se observa una tendencia creciente de los ingresos hasta cierto grupo de edad, luego del cual declinan.

Las tasas de retorno resultantes se equiparan dentro del promedio para estudios realizados dentro y fuera del país. Así, para la educación, la tasa promedio por año del retorno es de $8 \%, \mathrm{y}$, por niveles, la más significativa es la superior con $11,6 \%$. Respecto a la tendencia regional que ha tenido el retorno a la educación se observa que el retorno a la educación media cae, mientras que el retorno a la educación superior aumenta.

Adicionalmente estos hechos se vinculan con el crecimiento en la medida que la tasa de escolaridad aumente $\mathrm{y}$, por consiguiente, aumentará la productividad de la fuerza laboral que es lo que causa el aumento de las retribuciones reales del trabajo. 
Para verificar que se estime correctamente el retorno a la educación, se utilizaron diversas especificaciones que permiten incorporar nuevas variables de control. Al enfrentar el problema cuando se estima con experiencia potencial, se utilizaron datos para experiencia efectiva en años y se observa que se sobrestimaba el retorno a la educación en dos puntos porcentuales. Cuando se divide en niveles educativos, se evidencia que esta disminución se debe especialmente a la caída en el retorno a la educación secundaria.

Al controlar por educación de los padres, resulta que el retorno a la educación se reduce en un punto porcentual. Controlando por calidad de educación y habilidades, utilizando proxies, se encuentra que el retorno la educación alcanza un 4,5\%, lo que motiva para seguir invirtiendo en educación.

Con el análisis de la descomposición simple de varianza podemos concluir que un tercio de la desigualdad de los salarios se atribuye a estos factores observables como educación y experiencia y el restante porcentaje de desigualdad a los demás factores que son inobservables.

La desigualdad salarial en el Ecuador, según el Banco Mundial (2014) a través del coeficiente de Gini, alcanza un 46,6\%, y la evolución de los salarios reales para distintos grupos demográficos de trabajadores experimenta importantes discrepancias. Con diferencias salariales crecientes por nivel educacional, siendo más beneficiosa en el caso de los hombres.

Finalmente, queda demostrado, en buena parte, que invertir en capital humano ampliando su nivel educativo o escolaridad formal no solo mejora la productividad de los agentes, sino también garantiza un retorno por cada año y nivel educativo que se diferencia conforme la estructura económica y productiva de cada localidad, ciudad o país; no obstante, la sociedad ecuatoriana actual debe mantener el incentivo por alcanzar el máximo nivel de educación y promover el crecimiento social y la calidad de vida de sus hogares y la sociedad en general.

\section{Referencias}

Angrist, Joshua, y Alan Krueger. 1991. "Does Compulsory School Atendance Affect Schooling and Earnings". The Quaterly Journal of Economics 106, No. 4: 979-1014.

---. 1992. Estimating the Payoff to Schooling Using the Vietnam-era Draft Lottery. Cambridge: National Bureau of Economic Research. 
Angrist, Joshua, y Whitney Newey. 1991. "Over Identification Test in Earnings Functions with Fixed Effects". Journal of Bussines \& Economics Statistics 9, No. 3: 317-323.

Ashenfelter, Orley, y Alan Krueger. 1994. "Estimates of the Economic Return to Schooling from a New Sample of Twins". The American Economic Review 84, No. 5: 1157-1173.

Becker, Gary. 1964. Human Capital. Nueva York: Columbia University Press.

Bravo, David, Dante Contreras y Tomas Rau. 1999. Wage Inequality and Labor Supply in Chile 19901996: Non Parametric Approach. Santiago: Departamento de Economía, Universidad de Chile.

Bravo, David, y Alejandra Marinovic. 1997. Wage Inequality in Chile: 40 Years of Evidence. Santiago: Departamento de Economía-Universidad de Chile.

Butcher, Kristin, y Anne Case. 1994. "The Effect of Sibling Sex Composition on Women's Education and Earnings". The Quaterly Journal of Economics 109, No. 3 (agosto): 531-563.

Card, David. 1999. "The Causal Effect of Education on Earnings". Handbook of Labor Economics, No. 3 (Berkeley: University of California): 1801-1863.

Contreras, Dante, David Bravo y Patricia Medrano. 1999. Measurement Error: Unobservables and Skill Bias in Estimating the Return to Education in Chile. Santiago: Departamento de Economía-Universidad de Chile.

Griliches, Zvi. 1977. "Estimating the Return to Schooling: Some Econometric Problems". Econométrica 45, No. 1: 1-22.

Heckman, James. 1979. "Sample Selection Bias as a Specification Error". Econometrica 47, No. 1: 153-161.

Instituto Nacional de Estadística y Censos (INEC). 1995. Encuesta de condiciones de vida. Dirección de Comunicación Social-INEC.

Katz, Lawrence, y Kevin Murphy. 1992. "Changes in Relative Wages, 1963-1987: Supply and Demand Factors”. The Quarterly Journal of Economics 107, No. 1: 35-78.

Lam, David, y Robert Schoeni. 1993. "Effects of Family Background on Earnings and Returns to Schooling: Evidence from Brazil”. Journal of Political Economy 101, No. 4: 710-740.

Mancero, Alfredo. 1997. Educación, ¿para qué? Quito: Corporación Editora Nacional.

Mincer, Jacob. 1958. "Investment in Human Capital and Personal Income Distribution". Journal of Political Economy 66, No. 4: 281-302.

Psacharopoulos, Jorge. 1980. Returns to Education: An Updated International Comparison. Londres: London School of Economics / World Bank.

---. 1985. "Returns to Education: A further International Update and Implications". Journal of Human Resources 20, No. 4: 583-604.

Samaniego, Pablo. 1995. "El ingreso y la educación en Ecuador: análisis por niveles de instrucción". (Quito: Cuestiones Económicas, Banco Central del Ecuador, No. 24, marzo 1995).

Schultz, Theodore W. 1961. "Investment in Human Capital". The American Economic Review 51, No. 1: 1-17.

The World Bank. Index Mundi. 2013. Consulta: enero de 2014. «http://data.worldbank.org/ indicator/SI.POV.GINI . 